\title{
A novel economical duty cycle division multiplexing with electrical multiplexer and demultiplexer for optical communication systems
}

\begin{abstract}
Duty cycle division multiplexing (DCDM) is proposed as an alternative multiplexing technique. In this technique, the channel multiplexing and demultiplexing are performed electrically. This technique allows aggregate bit rate to be recovered at the single channel bit rate, which is very economic. In this paper, we examine three channels system where each channel operates at $10 \mathrm{~Gb} / \mathrm{s}$ over a single optical carrier. Performance of the system is evaluated based on back-to-back receiver sensitivity, optical signal-to-noise ratio (OSNR) and chromatic dispersion tolerance. The performance comparison is made against return-tozero (RZ) format. The results show that, DCDM can support higher amount of chromatic dispersion than that RZ. At $3 \times 40 \mathrm{~Gb} / \mathrm{s}$, a receiver sensitivity and OSNR of $-16.8 \mathrm{dBm}$ and $34.6 \mathrm{~dB}$ is respectively required for the worst DCDM channel.
\end{abstract}

Keyword: Optical communications; Duty cycle division multiplexing; DCDM; Electrical multiplexers; Channel demultiplexing; Economical transceivers; Return-to-zero; Chromatic dispersion; Receiver sensitivity; Optical signal-to-noise ratio; OSNR; Clock recovery; Data recovery 